\title{
Effect of Aerobic Dance on Cardiorespiratory Fitness in Pregnant Women: A Randomised Controlled Trial
}

\author{
Nayela Sayeed $^{1}$, W. Nagadeepa ${ }^{2}$, Vemulapalli Ravi Kuma ${ }^{3}$, Penjuri Subhash Chandra Bose ${ }^{4}$ \\ ${ }^{1}$ Assistant Professor, Physiology, Shadan Institute of Medical Sciences, Telangana. Professor, Physiology, \\ MNRMC, Fasalwadi, Sangareddy, Telangana-502294, '2 Professor Physiology MNRMC, Fasalwadi, Sangareddy, \\ Telangana-502294, ${ }^{3}$ Associate Professor, Dept. of Pharmacology, MNR College of pharmacy, Fasalwadi, \\ Sangareddy, Telangana-502294, ${ }^{4}$ Professor Dept. of Pharmaceutics, MNR College of pharmacy, Fasalwadi, \\ Sangareddy, Telangana -502294.
}

\begin{abstract}
Introduction: It is recommended that healthy pregnant women should undertake moderate physical activity for 30 minutes on most days of the week throughout pregnancy ${ }^{1,4}$. The aim of the present study was to evaluate the effect of a 12-week programme of twice-weekly aerobic dance classes, in addition to 30 minutes of moderate self-imposed physicalactivity on the remaining week days, on cardiorespiratory fitness in primiparous women.
\end{abstract}

Material and Method: This study was a secondary analysis of a randomized controlledtrial comparing a group undertaking aerobic exercise with a non-exercising control group.

Results and Conclusion: This study showed that a 12-week aerobic dance programme had no effect on cardiorespiratory fitness in pregnant women. It has been reported that even small improvements in cardiorespiratory fitness may cause an overall lower mortality rate in adults ${ }^{24,25}$.

Keywords: Aerobic Dance, Cardiorespiratory Fitness, Pregnancy.

\section{Introduction}

It is recommended that healthy pregnant women should undertake moderate physical activity for 30 minutes on most days of the week throughout pregnancy ${ }^{1-4}$. Observational studies have shown that pregnant women have a low level of physical activity, and few women exercise on a regular basis ${ }^{5-7}$. It is known that physical fitness is more important than the level of physical activity for the achievement of health benefits in the general population ${ }^{8-10}$. As such, the focus during pregnancy should be to maintain physical fitness

\footnotetext{
Corresponding Author:

Dr. W. Nagadeepa

Professor Physiology MNRMC, Fasalwadi,

Sangareddy, Telangana -502294

e-mail: drdeeparamesh6@gmail.com
}

A Cochrane review ${ }^{11}$ concluded that regular exercise during pregnancy seems to improve or maintain cardiorespiratory fitness. However, the authors stated that the studies were generally of low methodological quality of the reference list and an additional search on PubMed (up to February 2010) only identified two randomized controlled trials of high methodological quality $^{12,13}$. Pregnancy leads to physiological and anatomical changes that may affect women's cardiorespiratory fitness. Firstly, weight gain leads to a progressive decline in performance ${ }^{14}$. Secondly, there is an increase in blood volume and heart rate ${ }^{15}$, the maximal heart rate is reduced and the blood has a lower concentration of hemoglobin during pregnancy ${ }^{14}$. Thirdly, minute ventilation increases by almost $50 \%{ }^{15}$. Hence, these changes lead to reduced reserve work capacity in pregnant women.

The aim of the present study was to evaluate the effect of a 12-week programme of twice-weekly aerobic 
dance classes, in addition to 30 minutes of moderate self-imposed physical activity on the remaining week days, on cardiorespiratory fitness in primiparous women

Material and Method: This study was a secondary analysis of a randomized controlled trial comparing a group undertaking aerobic exercise with a nonexercising control group The study was conducted in the Physiology Department, MNR Medical College, Sangareddy. In total, 105 women were randomized to either the exercise group $(n=52)$ or the control group $(\mathrm{n}=53)$. of these, 62 women (exercise group, $\mathrm{n}=34$; control group, $\mathrm{n}=28$ ) completed baseline and postintervention fitness.

Table 1: Aerobic dance exercise programme.

\begin{tabular}{|c|c|c|}
\hline Time & Part & Intensity \\
\hline 5 minutes & Warm-up & \\
\hline 35 minutes & Aerobic dance & $\begin{array}{l}12 \text { to } 14 \text { (somewhat hard) } \\
\text { on the Borg scale }\end{array}$ \\
\hline 15 minutes & Muscle Exercises & $\begin{array}{l}12 \text { to } 15 \text { repetitions, } \\
\text { maximum three sets }\end{array}$ \\
\hline
\end{tabular}

Inclusion Criteria: Healthy primiparous women who had not participated in a structured exercise programme ( $>60$ minutes once per week), including brisk walking ( $>120$ minutes per week), during the past 6 months were eligible for the trial. Other inclusion criteria were gestational age of 12 to 24 weeks, and able to read and understand instructions. Those subjects who were willing to participate in the study were included after obtaining informed consent.

\section{Exclusion Criteria:}

Included: Severe heart disease, pregnancy- induced hypertension, history of two or more miscarriages, bleeding after 12 weeks of gestation, uncontrolled thyroid disease, preeclampsia, or other diseases that could interfere with participation ${ }^{1}$.

\section{Study Design: Randomized controlled trial}

Statistical Analysis: Background variables are presented as means with standard deviations (SD) and frequencies (\%).between the groups at baseline were examined using a two- sided independent sample t-test for continuous variables, and Chi-squared test for categorical variables ${ }^{16}$. The principal analysis was based on participants who had completed both baseline and post-intervention fitness tests (exercise group, $\mathrm{n}$ $=34$; control group, $\mathrm{n}=28$ ). In addition, per-protocol analysis was undertaken based on participants with $80 \%$ adherence to the exercise protocol (19 exercise classes: exercise group, $\mathrm{n}=18$; control group, $\mathrm{n}=28$ ). The women in the exercise group were significantly older than the women in the control group; therefore, analysis of covariance was used to examine the difference in change in cardiorespiratory fitness between the groups ${ }^{17}$. The post-intervention score for cardiorespiratory fitness was set as the dependent variable, and baseline score and age were set as covariates ${ }^{18}$. Statistical analyses were conducted in Statistical Package for the Social Sciences Version 18 (SPSS Inc., Chicago, IL, USA), and the level of statistical significance was set at $\mathrm{P}<0.05$.

\section{Results}

In total, 105 women were randomized to either the exercise group $(n=52)$ or the control group $(n=53)$. of these, 62 women (exercise group, $\mathrm{n}=34$; control group, $\mathrm{n}=28$ ) completed baseline and post-intervention fitness tests. Fig. 1 (see online supplementary data) shows the flow chart of participants. Since there was a high dropout rate, only women who performed both fitness tests were included in the analyses ${ }^{19}$.

The participants' personal characteristics are shown in Table 2. Women in the exercise group were significantly older $(\mathrm{P}=0.03)$ than

Table 2: Personal characteristics at baseline in the exercise $(n=34)$ and control $(n=28)$ groups.

\begin{tabular}{|l|c|c|}
\hline Characteristic & $\begin{array}{c}\text { Exercise } \\
\boldsymbol{n}=\mathbf{3 4}\end{array}$ & $\begin{array}{c}\text { Control } \\
\boldsymbol{n}=\mathbf{2 8}\end{array}$ \\
\hline Age (years), mean (SD) & $31.5(3.2)$ & $29.5(4.0)$ \\
\hline Gestational week mean (SD) & $17.1(3.9)$ & $18.5(4.4)$ \\
\hline Height (m), mean (SD) & $1.68(0.1)$ & $1.70(0.1)$ \\
\hline Pre-pregnancy weight (kg), mean (SD) & $64.9(9.5)$ & $66.4(8.4)$ \\
\hline Weight (kg) a, mean (SD) & $68.9(9.9)$ & $71.3(8.2)$ \\
\hline $\begin{array}{l}\text { Pre-pregnancy body mass index } \\
\left(\mathrm{kg} / \mathrm{m}^{2}\right), \text { mean (SD) }\end{array}$ & $22.9(3.2)$ & $23.0(2.9)$ \\
\hline Married/living together, $n(\%)$ & $33(97.1)$ & $28(100)$ \\
\hline Daily smoker, $n(\%)$ & $1(2.9)$ & $0(0)$ \\
\hline
\end{tabular}

SD, Standard Deviation

a Measured at baseline.

Women in the control group, Apart from age, there were no significant differences in background variables between the two groups at baseline ${ }^{20}$. Furthermore, no significant differences in measurement of the outcome variables between the groups were found at baseline (Table 3). 
Table 3: Oxygen uptake, work load, heart rate and rating of perceived exertion (RPE) at Levels 1, 2 and 3 in the exercise and control groups at baseline and after the intervention, and mean adjusted difference in change between the groups ${ }^{21}$.

\begin{tabular}{|c|c|c|c|}
\hline Variable & Group & No. of Participants $(n)$ & $P$-value of difference in change \\
\hline \multicolumn{4}{|c|}{ VO2 (ml/kg/minute) } \\
\hline \multirow{2}{*}{ Level 1} & Exercise & 34 & \multirow{2}{*}{0.48} \\
\hline & Control & 25 & \\
\hline \multirow{2}{*}{ Level 2} & Exercise & 31 & \multirow{2}{*}{0.98} \\
\hline & Control & 24 & \\
\hline \multirow{2}{*}{ Level 3} & Exercise & 24 & \multirow{2}{*}{0.89} \\
\hline & Control & 19 & \\
\hline \multicolumn{4}{|c|}{ Work load (inclination \%) } \\
\hline \multirow{2}{*}{ Level 1} & Exercise & 33 & \multirow{2}{*}{0.14} \\
\hline & Control & 28 & \\
\hline \multirow{2}{*}{ Level 2} & Exercise & 32 & \multirow{2}{*}{0.67} \\
\hline & Control & 27 & \\
\hline \multirow{2}{*}{ Level 3} & Exercise & 26 & \multirow{2}{*}{0.22} \\
\hline & Control & 21 & \\
\hline \multicolumn{4}{|c|}{ Heart rate (beats/minute) } \\
\hline \multirow{2}{*}{ Level 1} & Exercise & 34 & \multirow{2}{*}{0.85} \\
\hline & Control & 28 & \\
\hline \multirow{2}{*}{ Level 2} & Exercise & 32 & \multirow{2}{*}{0.52} \\
\hline & Control & 27 & \\
\hline \multirow{2}{*}{ Level 3} & Exercise & 26 & \multirow{2}{*}{0.23} \\
\hline & Control & 21 & \\
\hline
\end{tabular}

The women in the exercise group attended a mean of 20 (SD 12) out of 24 aerobic dance classes. Eighteen of 34 women $(53 \%)$ in the exercise group completed the prescribed exercise, protocol (80\%) with 19 aerobic dance classes. Thirty-two of 34 women (94\%) in the exercise group returned their exercise diaries. In addition to the aerobic dance classes, women in the exercise group reported a mean weekly exercise time of 90 (SD 73) minutes in their exercise diaries ${ }^{22}$. Walking was the most common form of exercise, followed by crosscountry skiing, biking, muscular strength training and swimming. No exercise-related injuries or other adverse events were reported by the participants in the exercise group. Two of 28 women (7\%) in the control group reported that they had exercised at a moderate level of intensity at least twice per week for a minimum of 60 minutes during the intervention period ${ }^{23}$.
Change in cardiorespiratory fitness: The numbers of participants in the analyses vary as some participants did not continue the test to Levels 2 and 3 and because of error during tests ${ }^{24}$.

Oxygen uptake: The differences in change in relative VO2 $(\mathrm{ml} / \mathrm{kg} /$ minute) between the groups were not significant at Level 1, 2 or 3 (Table 3). Nine of 34 $(26 \%)$ women in the exercise group had an increase in $\mathrm{VO} 2(\mathrm{ml} / \mathrm{kg} /$ minute) after the intervention, compared with five of $25(20 \%)$ women in the control group (P = 0.56). Furthermore, there were no significant differences in change in absolute $\mathrm{VO} 2(\mathrm{l} /$ minute) between the groups at any level ${ }^{25}$.

Work load: There were no significant differences between the groups in change in work load at Level 1, 2 or 3 (Table 3 ). Four of 33 (12\%) women in the exercise 
group walked at a higher inclination before onset of blood lactate accumulation after the intervention, compared with only one of $28(4 \%)$ women in the control group $(\mathrm{P}=0.36)^{26}$.

Heart rate: There were no significant differences between the groups in change in heart rate at Level 1, 2 or 3 (Table 3). Both groups had a lower heart rate at onset of blood lactate accumulation after the intervention, indicating a lower intensity at the same blood lactate level. There were no differences in the proportion of women with a higher heart rate before onset of blood lactate accumulation after the intervention between the groups $(\mathrm{P}=0.88)^{27}$.

Rating of perceived exertion: The differences between the groups in change in RPE on the Borg scale were not significant at Level 1, 2 or 3 (Table 3). Nineteen of $34(56 \%)$ women in the exercise group and 10 of 28 (36\%) women in the control group reported a lower RPE during the post-intervention fitness test $(\mathrm{P}=0.13)$.

Per-protocol analyses (data not shown) based on participants with $\geq 80 \%$ adherence to the exercise protocol (exercise group, $\mathrm{n}=18$; control group, $\mathrm{n}=28$ ) did not change any of the above mentioned results ${ }^{28}$.

\section{Conclusion}

A 12-week aerobic dance programme had no effect on cardiorespiratory fitness in pregnant women. Further randomized controlled trials of high methodological and interventional quality on the effect of regular exercise on cardiorespiratory fitness in sedentary pregnant women are warranted.

\section{Conflict of Interest: Nil}

\section{Source of Funding- Self}

Ethical Clearance: It was given by the institution.

\section{References}

1. ACOG Committee Opinion. Exercise during pregnancy and the post- partum period. Number 267, January 2002. American College of Obstetriciansand Gynecologists. IntJ Gynaecol Obstet 2002;77:79-81.

2. Sport Medicine Australia. SMA statement. The benefits and risks of exercise during pregnancy. J Sci Med Sport 2002;5:11-9.

3. Wolfe LA, Davies GA. Canadian guidelines for exercise in pregnancy. Clin Obstet Gynecol 2003;46:488-95.

4. Royal College of Obstetriciansand Gynaecologists (RCOG).Exercise in pregnancy. RCOG statement 42006.

5. Evenson KR, Savitz DA, Huston SL. Leisure-time physical activ- ity among pregnant women in the US. Paediatr Perinat Epidemiol 2004;18:400-7.

6. Haakstad LA, VoldnerN, Henriksen T, Bo K. Physical activity level and weight gain in a cohort of pregnant Norwegian women. Acta Obstet Gynecol Scand 2007;86:559-64.

7. Owe KM, Nystad W, BoK. Correlates of regular exercise during pregnancy: the Norwegian Mother and Child Cohort Study. Scand J Med Sci Sports 2008;19:637-45.

8. Lee DC, SuiX, Ortega FB, KimYS, ChurchTS, Winett RA, etal.Com- parisons of leisure-time physical activity and cardiorespiratory fitness as predictors of all-cause mortality in men and women. BrJ Sports Med 2011;45:504-10.

9. Sassen B, Cornelissen VA, Kiers H, Wittink H, Kok G, Vanhees L. Physical fitness matters more than physical activity in controlling cardiovascular disease risk factors. Eur J Cardiovasc PrevRehabil 2009; 16:677-83.

10. Fogelholm M. Physicalactivity, fitness and fatness: relations to mortality, morbidity and disease risk factors. A systematic review. Obes Rev 2010;11:202-21.

11. Kramer MS, McDonald SW.Aerobicexerciseforw omenduring pregnancy. Cochrane Database Syst Rev 2009;4:CD000180.

12. Santos IA, Stein R, Fuchs SC, Duncan BB, Ribeiro JP, Kroeff LR, etal. Aerobic exercise and submaximal functional capacity in overweight pregnant women: a randomized trial. Obstet Gynecol 2005;106:243-9.

13. BaciukEP, Pereira RI, Cecatti JG, Braga AF, Cavalcante SR. Water aerobics in pregnancy: cardiovascular response, labor and neonatal outcomes. Reprod Health 2008;5:10.

14. Wolfe LA, Charlesworth SA, Glenn NM, Heenan AP, Davies GA. Effects of pregnancy on maternal work tolerance. Can J Appl Physiol 2005;30:21232.

15. Artal R, O'Toole M. Guidelines of the American 
College of Obstetricians and Gynecologists for exercise during pregnancy and the postpartum period. Br J Sports Med 2003;37:6-12.

16. Collings CA, Curet LB, Mullin JP. Maternal and fetal responses to a maternal aerobic exercise program. Am J Obstet Gynecol 1983;145:702-7.

17. Erkkola R. The influence of physical training during pregnancy on physical work capacity and circulatory parameters. Scand J Clin Lab Invest 1976;36:747-54.

18. Lynch AM, McDonald S, MagannEF, Evans SF, Choy PL, Dawson B, etal. Effectiveness and safety of a structured swimming program in previously sedentary women during pregnancy. J Matern Fetal Neonatal Med 2003;14:163-9.

19. South-Paul JE, Rajagopal KR, Tenholder MF. The effect of participation in a regular exercise program upon aerobic capacity during pregnancy. Obstet Gynecol 1988;71:175-9.

20. Kardel KR. Effects of intense training during and after pregnancy in top-level athletes. Scand J Med Sci Sports 2005;15:79-86.

21. Borg G. Perceived exertion as an indicator of somatic stress. Scand J Rehabil Med 1970;2:92-8.

22. Jones $\mathrm{AM}$, Carter $\mathrm{H}$. The effect of endurance training on parameters of aerobic fitness. Sports Med 2000;29:373-86.
23. Spurway N, Jones AM. Lactate testing. In: Winter EM, Jones AM, Davison RCR, Bromley PD, Mercer TH, editors. Sport and exercise physiology testing: guidelines. The British Association of Sport and Exercise Sciences guide. Great Britain: Routledge; 2007. p.112-9.

24. MyersJ, Prakash M, Froelicher V, DoD, Partington S, Atwood JE. Exercise capacity and mortality among men referred for exercise testing. N Engl J Med 2002;346:793-801.

25. Keteyian SJ, Brawner CA, Savage PD, Ehrman JK, Schairer J, Divine G, etal. Peak aerobic capacity predicts prognosis inpatients with coronary heart disease. Am Heart J 2008;156:292-300.

26. Bosquet L, Leger L, Legros P. Method to determine aerobic endurance. Sports Med 2002;32:675-700.

27. Armijo-Olivo SWS, Magee D. Intentiontotreat analysis, compliance, drop-outs an how to deal with missing data in clinical research: are view. Phys Ther Rev 2009; 14:36-49.

28. Garber CE, Blissmer B, Deschenes MR, Franklin BA, La Monte MJ, Lee IM, et al. American College of Sports Medicine position stand. Quantity and quality of exercise for developing and maintaining cardiorespiratory, musculoskeletal, and neuromotor fitness in apparently healthy adults: guidance for prescribing exercise. MedSci Sports Exerc 2011;43: 